\title{
ACHim Aurnhammer
}

Martin Opitz، petrarkistisches Mustersonett Francisci Petrarchae (Canzoniere 132), seine Vorläufer und Wirkung 


\section{Martin Opitz' petrarkistisches Mustersonett Francisci Petrarchae (Canzoniere 132), seine Vorläufer und Wirkung}

\section{Vorgeschichte der Übersetzung}

Um 1625 ist die deutsch-lateinische Sprachenkonkurrenz in Deutschland zugunsten einer nationalsprachlichen Dichtung entschieden. ${ }^{1}$ Doch noch um 1600 stand in Deutschland die Option der ästhetischen Elite für ein Dichten in deutscher Sprache keineswegs fest. Denn die Späthumanisten suchten die Modernität des Neulatein dadurch zu erweisen, daß sie die zeitgemäßen volkssprachlichen Formen wie Tragikomödie, Romanzo oder Sonett lateinisch imitierten und assimilierten. $^{2}$

Auch die Einbürgerung des Sonetts und des petrarkistischen Diskurses in Deutschland ist von diesem Wettstreit geprägt: So zitiert Opitz in seinem Aristarchus (1617) die formgetreue Verdeutschung von Petrarcas Proömialsonett des Ernst Schwabe von der Heide, um die Konkurrenzfähigkeit des Deutschen als Literatursprache zu verbürgen. Demgegenüber stehen - von neulateinischen Originalsonetten abgesehen - Übersetzungen von Sonetten Petrarcas ins Lateinische. Weil Petrarca als lateinischer Moralphilosoph bei den Späthumanisten allgemein geschätzt war, erprobte man die Innovationsfähigkeit des Neulateinischen gern an seinen italienischen Dichtungen, vor allem an dem Sonett 132 aus dem Canzoniere:

1 Das Jahr 1617 markiert eine Epoche in der deutschen Literaturgeschichte: die »Fruchtbringende Gesellschaft" wird gegründet, und Martin Opitz veröffentlicht den Aristarchus sive de contemptu linguae Teutonicae. Während Fürst Ludwig von Anhalt-Köthen die Förderung der deutschen Sprache zu einem Anliegen fürstlicher Kulturpolitik machte, suchte Opitz in seinem lateinischen Aristarchus den späthumanistischen Gelehrtenstand davon zu überzeugen, daß Deutsch eine konkurrenzfähige Literatursprache sei.

2 So wurden in Deutschland die modernen italienischen Tragikomödien (Tasso, Guarini) und Anstandslehren bezeichnenderweise zuerst in lateinischer Sprache assimiliert; vgl. Vf.: Andreas Hiltebrand - ein pommerscher Dichterarzt zwischen Späthumanismus und Frühbarock. In: Pommern in der Frühen Neuzeit. Literatur und Kultur in Stadt und Region. Hg. von Wilhelm Kühlmann und Horst Langer. Tübingen 1994 (Frühe Neuzeit 19), S. 199225. Die neulateinische Sonettistik um 1600 ist bislang nur lückenhaft erforscht. Einige Hinweise dazu bei Walter Mönch: Das Sonett. Gestalt und Geschichte. Heidelberg 1955, S. 140f. und S. 145, Francesco Novati: Sonetti latini e semilatini nel Trecento. In: Studi Medievali 2 (1906/07), S. 109-112, Jozef Ijsewijn und Dirk Sacré: Companion to NeoLatin Studies, Part II: Literary, Linguistic, Philological and Editorial Questions. Leuven ${ }^{2}$ 1998, S. 15-20, und Elena Maria Duso: Il sonetto latino e semilatino in Italia nel Medioevo e nel Rinascinento. Rom 2004. 


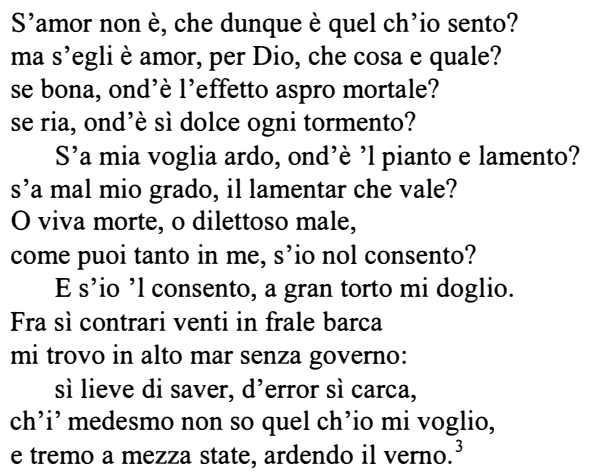

Dieses Sonett ist bekannt wegen der suggestiven Reihe dilemmatischer Liebesfragen, die den Aufgesang prägen. ${ }^{4}$ In seiner mehrgliedrigen Dualität und Antithetik ist es freilich weniger typisch für den Canzoniere, als vielmehr für den Petrarkismus, da es dessen komplexe Pluralität und stilistische Komplikation antizipiert. ${ }^{5}$ Als Martin Opitz um 1620 das Sonett Francisci Petrarchoe erstmals

3 Petrarca: Canz. 132. Zum besseren Verständnis diene die relativ textnahe Vers-Übertragung:

Ists Liebe nicht: was ists, was ich empfinde?

Ists Liebe - Gott, welch Ding? von welchem Schlage?

wenn gut: woher dann Tod und Trauertage?

wenn schlecht: woher dann jede Qual so linde?

Brenn ich mit Fleiß: hat dann die Klage Gründe?

wenn wider Willen, dann - was nützt die Klage?

lebendiger Tod, o angenehme Plage,

wieso vermögt ihr, was ich unterbinde?

Und stimm ich zu, ists Unrecht, daß ich klage!

Inmitten solcher Widerwinde finde

ich mich auf hohen Wogen ohne Steuer:

so leicht an Wissen, so beschwert mit Sünde,

daß ich, zu deuten was ich will, verzage:

im Sommer zitternd spür ich winters Feuer.

Canzoniere nach einer Interlinearübersetzung von Geraldine Gabor in deutsche Verse gebracht von Ernst-Jürgen Dreyer mit Anmerkungen zu den Gedichten von Geraldine Gabor. Basel und Frankfurt/M. ${ }^{2} 1989$, S. 393.

4 Die Forschung führt die Fragetechnik nach dem Muster des 'Sic et non zurück; vgl. Ulrich Maché: Die Unbegreiflichkeit der Liebe. Das Petrarca-Sonett von Martin Opitz. In: Gedichte und Interpretationen. Bd. 1: Renaissance und Barock. Hg. von Volker Meid. Stuttgart 1982, S. 125-135, hier 130. Das Sonett folgt aber nicht nur der scholastischen Formel der >quaestio<, sondern auch dem kanonischen Argumentationsmuster >quaestio an sit‘; eine genaue Analyse der >quaestiones` in Petrarcas Sonett bietet Piero Boitani: Il tragico e il sublime nella letteratura medievale. Bologna 1992 [engl. OF 1989], S. 93-99.

5 Vgl. dazu Dámaso Alonso: Die Dichtung Petrarcas und der Petrarkismus. (Zur Ästhetik der Pluralität). In: Übersetzung und Nachahmung im europäischen Petrarkismus. Studien und Texte. Hg. von Luzius Keller. Stuttgart 1974 (Studien zur Allgemeinen und Vergleichenden Literaturwissenschaft 7), S. 104-154. Die Antinomie gehört zu den wenigen unstrittigen Merkmalen des petrarkistischen Systems; vgl. Hans Pyritz: Petrarca und die deutsche 
ins Deutsche übersetzte, gehörte es längst zu den direkten Vorbildern der europäischen Petrarkisten. ${ }^{6}$ Neben Übertragungen ins Englische, Spanische und Französische ist das Sonett - öfter als in jede andere Sprache - mindestens fünfmal ins Neulateinische übersetzt worden. ${ }^{?}$

\section{Neulateinische Versionen vor Opitz}

Die neulateinischen Versionen von Coluccio Salutati, Cristoforo Landino, Thomas Watson, Wilhelm Canter und Georgius Tilenus zeichnen sich allesamt durch ästhetische Virtuosität aus. ${ }^{8}$ Syntaktisch kommen sie dem italienischen

Liebeslyrik des 17. Jahrhunderts. In: H. P.: Schriften zur deutschen Literaturgeschichte. Hg. von Ilse Pyritz. Köln 1962, S. 54-72, hier S. 58, und Klaus W. Hempfer: Probleme der Bestimmung des Petrarkismus. Überlegungen zum Forschungsstand. In: Die Pluralität der Welten. Aspekte der Renaissance in der Romania. Hg. von Wolf-Dieter Stempel und Karlheinz Stierle. München 1987, S. 253-277, bes. 265.

6 Keller (Hg.): Übersetzung und Nachahmung (Anm. 5), S. 314-333, hat allein vierzehn Übersetzungen und Nachahmungen in verschiedene europäische Sprachen zusammengestellt.

7 Vgl. Ernest Hatch Wilkins: The Making of the »Canzoniere« and Other Petrarchan Studies. Rom 1951, S. 293.

8 Vgl. Coluccio Salutati: „Si fors non sit amor « [wohl noch zu Lebzeiten Petrarcas]. In: Wilkins (Anm. 7), S. 262, Christophorus Landinus: Quaerit qua perturbatione sit affectus (Inc.: "Si non vexat amor« [6 Distichen]). In: Ch. L.: (Xandrae libri tres, I 14 [um 1443]) Carmina omnia. Florenz 1939, S. 16, Thomas Watson: »Hoc si non sit amor«. In: Hekatompathia 6 (1582), S. 43; wieder in: Keller (Anm. 6), S. 332. Die Version von Guglielmus Canter hat Opitz wohl gekannt:

De Amore. ex Petrarcha.

Quod si nil Amor est; quid me sentire putabo?

Sin aliquid; quaeso quale quid esse potest?

Si bonus; unde malos effectus credis oriri.

Sin malus; unde ferax copia mellis adest.

Sive volens uror; quorsum haec lamenta dolorque?

Sive aliter; nunquid plaugere profuerit?

O mors viva, malum non illae tabile, sive

Non consensus adest, quomodo tanta potes?

Sin consensus adest, non recte conqueror amens:

Sub geminis ventis in rate perfragili

Dorsa maris rectoris egens incerta peragro,

Plenus et erroris, menteque tam vacuus;

Ut non quid cupiam, detur mihi scire facultas, Ipsa aestate tremens, ustus et ipsa hieme.

[>Wenn Liebe nichts ist, was fühle ich dann? Wenn sie aber etwas ist, was, frage ich, kann es sein? Wenn sie gut ist, woher kommen die schlimmen Wirkungen? Wenn aber nicht schlimm, warum gibt es dann solch reiche Fülle an Süße? Wenn ich willentlich brenne, wozu dann Jammer und Schmerz? Wenn nicht, nützte es etwa zu klagen? O lebendiger Tod, nicht unerfreuliches Übel, wenn es kein Einverständnis gibt, wie vermagst du so viel? Wenn es aber Einverständnis gibt, zu Unrecht klage ich dann außer mir: Unter zweifachen Winden in schwankem Kahn durchstreife ich ohne Steuer die ungewisse hohe See, so reich 
Original nahe, sprachlich-stilistisch überbieten sie nicht selten sogar Petrarcas Sonett. Vor allem wird die plurale Antithetik forciert und paronomastisch intensiviert (»amarus Amor « [Landinus]).

Defizitär bleibt freilich durchweg die metrische Assimilation. Dieses Manko gilt nicht nur für die Übersetzungen in elegischem Versmaß eines Cristoforo Landino, Wilhelm Canter oder Georgius Tilenus, sondern auch für die versgetreuen Hexameter-Übertragungen von Coluccio Salutati und Thomas Watson, welche die 14 Verse in Sonettform anordnen: Alle neulateinischen Versionen ermangeln des Reims, der als zusätzlicher Bedeutungsträger den entscheidenden Vorzug der modernen Literatursprachen gegenüber den Klassischen Sprachen bildet.

Inwieweit die Latinisierungen sich dem italienischen Original Petrarcas anpassen, zeigt exemplarisch die Version des Georg Tilenus. Opitz dürfte sie gewiß gekannt haben, da sein Heidelberger Freund Janus Gruter sie ebenso wie Wilhelm Canters Version in seine neulateinische Anthologie, die Delitiae Poetarum Germanorum (1612), aufnahm:

\author{
Ex Italico Petrarchae \\ Si non est id amor, quid erit quod pectore fervet? \\ Sin amor est, qualis dic mihi, res sit amor? \\ Si bona, cur fructus acidos, cur funera gignit? \\ Sin mala: cur suave est tormina tanta pati? \\ Si cremor igne volens, quei lumina fletibus hument? \\ Sin patior vim, quid flere querique iuvat? \\ $\mathrm{O}$ mors viua, malum ô dulce, in mea pectora tantum \\ Te licet, assensu non sequar, vnde potes? \\ Sin sequar: iniusto memet male torqueo luctu, \\ Sic rotat oppositis me ratis aegra Notis. \\ Ante pedes avidum mare: sed moderamina clavi \\ Deserit ars: error pro duce vela regit. \\ Ipse, quid in votis mihi nescio debeat esse: \\ Frigore Sol medio me premit, igne gelu. ${ }^{9}$
}

Tilenus imitiert nicht nur Petrarcas syntaktische Struktur des Aufgesangs, indem er die sieben Fragesätze aus dem Oktett übernimmt, sondern sucht dem italienischen Prätext auch stilistisch nahezukommen. So imitiert Tilenus die charakteristische »Si«-Anapher, ahmt die Oxymora in den Versen 7 und 14 nach und intensiviert die lexikalische Verdichtung im Oktett (»Mortale« und »morte«,

an Wahn wie arm an Einsicht, so daß mir nicht die Fähigkeit gegeben ist zu wissen, was ich wünsche. Sogar im Sommer friere ich und brenne sogar im Winter.1]

In: Delitiae c. poetarum Belgicorum huius superiorisque aevi illustrium. Prima Pars. Hg. von Ranutius Gherus [d. i. Janus Gruter]. Frankfurt/M. (N. Hoffmann für I. Fischer) 1614, S. 946f. - Zur Version von Tilenus vgl. Anm. 9.

9 Georgius Tilenus: Ex Italico Petrarchae: In: Delitiae Poetarum Germanorum huius Superiorisque Aevi Illustrium. Pars VI. Hg. von Ranutius Gherus [d. i. Janus Gruter]. Frankfurt/M. (N. Hoffmann für I. Fischer) 1612, S. 873 [s. v. »Epigrammata《]; wieder in JörgUlrich Fechner (Hg.): Das deutsche Sonett. Dichtungen, Gattungspoetik, Dokumente. München 1969, S. 47. 
»lamento« und »lamentar«) durch je eine zusätzliche 〉Feuer८- (»fervet« [V. 1]) und >Herz - Korrelation (»pectore« [V. 1] und »pectora« [V. 7]). Um die Hexameter, die länger als Endecasillabi sind, aufzufüllen, bedient sich Tilenus gebräuchlicher Topoi und Wendungen des klassischen Latein. So konkretisiert Tilenus die Zwillingsformel in Petrarcas Fragesatz (»ond'è 'l pianto e lamento«) durch eine Verbmetapher (»quei lumina fletibus hument«), die Tibull nachgebildet ist. ${ }^{10}$ Oder er ergänzt das einfache Nomen »mare « durch die Adjektivmetapher "avidum" zu einer Horazischen Wendung. " Selbst die originelle Paromoiose in Vers 10, die das hilflose Drehen des Nachens im feindlichen Wind klanglich abbildet (»Sic rotat oppositis me ratis aegra Notis«), ist durch »Notus«, den poetischen `Südwind,, dem klassischen Latein verhaftet. Da auch Petrarcas religiöse Formel »per Dio" weggelassen ist, scheinen solche Latinismen dem Übersetzungsprinzip einer antikisierenden Klassizität geschuldet. Auch in seiner klassizistischen Metrik unterscheidet sich die neulateinische Version wesentlich von dem italienischen Original. Denn das elegische Versmaß unterläuft die Sonettform. Während die Wiedergabe der Quartette durch jeweils zwei Distichen noch adäquat scheint, widersprechen die drei Distichen der Zweigliederung des Abgesangs in zwei Terzette. Denn der sonettypische Zusammenhang des Sextetts geht verloren, da die Distichen metrische wie syntaktische Einheiten bilden. Tilenus überträgt Petrarcas italienisches Sonett in ein wirkungsvolles lateinisches Epigramm. Dementsprechend isoliert und verschärft das abschließende Distichon Petrarcas Antimetabole zur epigrammatischen Pointe.

\section{Die Opitzische Version Francisci Petrarchae}

Mit seiner Eindeutschung wollte Opitz sowohl den petrarkistischen Nachholbedarf in Deutschland mildern als auch den neulateinischen Petrarkismus überbieten, um die Akzeptanz des Dichtens in der Muttersprache unter den lateinischen Gelehrten zu verbessern. So beruft sich Opitz in der Vorrede zu den 1624 veröffentlichten Teutschen Poemata, wo sein Sonnet Auß dem Italienischen Petrarchae zuerst erschien, denn auch vorrangig auf Petrarca als maßgeblichen Kronzeugen, um für eine »Poësie in unserer Muttersprach « und deren Überlegenheit gegenüber dem Latein zu werben: »Der Sinnreiche Petrarcha hat mehr Lob durch sein Toscanisch erjaget, als durch alles das, was er sonsten jemahls geschrieben «. ${ }^{12}$

10 Tibull: Elegien I, 8, 68 (»Et tua iam fletu lumina fessa tument«).

11 Carmina I, 28, 18.

12 Martin Opitz: An den Leser. In: M. O. Teutsche Poemata. Abdruck der Ausgabe von 1624 mit den Varianten der Einzeldrucke und der späteren Ausgaben. Hg. von Georg Witkowski. Halle 1902 (Neudrucke deutscher Litteraturwerke 189-192), S. 5-9, hier 5. Hier folgt Opitz freilich ganz dem Plädoyer für die muttersprachliche Dichtung aus der Vorrede des Herausgebers Petrus Scriverius der Nederduytschen Poemata von Daniel Heinsius (1618): 


\section{Francisci Petrarchae}

ISt Liebe lauter nichts/ wie daß sie mich entzündet? Ist sie dann gleichwol was/ wem ist ihr Thun bewust? Ist sie auch gut vnd recht/ wie bringt sie böse Lust?

Ist sie nicht gut/ wie daß man Frewd' aus jhr empfindet?

Lieb' ich ohn allen Zwang/ wie kan ich Schmertzen tragen?

Muß ich es thun/ was hilfft's/ daß ich solch Trawren führ'?

Heb' ich es vngern an/ wer dann befihlt es mir?

Thue ich es aber gern'/ vmb was hab' ich zu klagen?

Ich wancke wie das Graß so von den kühlen Winden Vmb Vesperzeit bald hin geneiget wird/ bald her. Ich walle wie ein Schiff das durch das wilde Meer

Von Wellen vmbgejagt nicht kan zu Rande finden.

Ich weis nicht was ich wil/ ich wil nicht was ich weis: Im Sommer ist mir kalt/ im Winter ist mir heiß. ${ }^{13}$

Wenn Martin Opitz um 1620 das Sonett Nr. 132 ins Deutsche übersetzt, konkurriert er somit nicht nur mit Petrarca, sondern auch mit dem neulateinischen Petrarkismus. Diese doppelte Orientierung an lateinischen wie muttersprachlichen Autoritäten lenkt die Opitzischen Nachahmungen antiker Muster ${ }^{14}$ prägt

»De gheleerde Petrarcha $[\ldots]$ heeft driemal grooter eer in sijn moeders tale behaelt, ende is veel meer bekent gheworden door zijn Toscaenschen sanck, als door alle het gheen dat hy aen den dach gebrocht heeft«. Auch im Buch von der Deutschen Poeterey (1624) betont Opitz, daß »bey den Italienern erst Petrarcha die Poeterey in seiner Muttersprache getrieben hat" (M. O.: Buch von der Deutschen Poeterey (1624). Studienausgabe. Hg. von Herbert Jaumann. Stuttgart 2002, S. 25).

13 Zitiert wird der autorisierte Druck von 1625 (M. O.: Acht Bücher Deutscher Poematum. Breslau 1625) nach der Kritischen Ausgabe: Martin Opitz: Gesammelte Werke. Hg. von George Schulz-Behrend. Bd. 2, 2. Stuttgart 1979 (BLVS 301), S. 703 . Wichtige Varianten des von Martin Opitz nicht autorisierten Erstdrucks: M. O.: Sonnet. Auß dem Italienischen Petrarchee. In: Teutsche Pöemata und Aristarchus. Straßburg 1624, S. 26f. (wieder in: M. O.: Teutsche Poemata [Anm. 12], S. 48 [Nr. 20]):

Titel Francisci Petrarchae] Sonnet/ Auß dem Italienischen Petrarchæ 3 gut vnd recht] recht vnd gut 4 Frewd' aus] Freudt auß 5 Lieb' ich ohn allen Zwang/ wie kan ich Schmertzen tragen?] Lieb ich gar williglich/ wie daß ich Schmertzen trage? 6 hilfft's] hilffts führ'?] führ? 7 Heb' ich es vngern an/ wer dann befihlt es mir?] Thue ichs nicht gern/ wer ists/ der es befihlet mir? 8 Thue ich es aber gern'/ vmb was hab' ich zu klagen?] Thue ich es gern/ warumb/ daß ich mich dann beklage? $9 \mathrm{Gra}]$ Gras 10 her.] her: 11 das durch das wilde] daß in dem wilden 13 weis] weiß wil] will

Die bisherigen Interpretationen des Sonetts von Janis Little Gellinek: Die weltliche Lyrik des Martin Opitz. Bern und München 1973, S. 101-103, Stephen Zon: Imitations Petrarch: Opitz, Fleming. In: Daphnis 7 (1978), S. 497-512, Maché (Anm. 4) und Fritz Wagner: Martin Opitz e Petrarca. In: Quaderni Petrarcheschi 7 (1990), S. 265-279, lassen die neulateinische Rezeption des Gedichts außer Acht. Lediglich Ulrich Schulz-Buschhaus: Emphase und Geometrie. Notizen zu Opitz' Sonettistik im Kontext des europäischen >Petrarkismus`. In: Martin Opitz (1597-1639): Nachahmungspoetik und Lebenswelt. Hg. von Thomas Borgstedt und Walter Schmitz. Tübingen 2002, S. 73-87, bedenkt die »Formkräfte des (neu)lateinischen Epigramms und der (neu)lateinischen Elegie« (S. 83).

14 Vgl. die exemplarische Studie von Wilhelm Kühlmann: »Militat omnis amans«. Petrarkistische Ovidimitatio und bürgerliches Epithalamion bei Martin Opitz. In: Daphnis 1 (1978), S. 199-214. Nach George Schoolfield: Propertius and Opitz: A compound footnote. In: 
aber auch seine übersetzerischen Aneignungen moderner volksspachlicher Dichtung, woraus eine charakteristische Mischung von »Emphase und Geometrie " resultiert. ${ }^{15}$ Die klassizistische Tendenz, in der Deutung des Gedichts bislang unberücksichtigt geblieben, zeigt sich deutlich im Tenor der PetrarcaÜbersetzung. Denn Opitz dämpft die Emphase des pathetischen Soliloquiums in der klassizistischen Manier der neulateinischen Übersetzer vor ihm: alle drei Apostrophen "per Dio« (V. 3) und »O viva morte, o dilettoso Male« fallen bei ihm weg. ${ }^{16}$ Mit der ersten Verdeutschung des prominenten und petrarkistischsten Petrarca-Sonetts verfolgte Opitz mehrere Ziele. Zum einen wollte er die lateinischen Versionen nachahmend überbieten, zum andern die deutsche Literatursprache formal wie thematisch modernisieren und drittens der zeitgemäßen Liebesrhetorik des Petrarkismus in Deutschland den Weg ebnen.

Die Eigenart der Opitzischen Übersetzung erhellt aus dem Vergleich mit dem italienischen Original. Petrarcas Endecasillabi gibt Opitz mit Alexandrinern wieder. Deren Zäsur in der Versmitte paßt Opitz geschickt den zweiteiligen Fragen an. Über das Versmaß hinaus ändert Opitz Petrarcas Sonettstruktur. Während Petrarca die Reime des ersten Quartetts im zweiten wiederholt, beginnt Opitz - entsprechend französischer Neuerungsversuche und entgegen seiner eigenen Sonett-Theorie ${ }^{17}$ - in Vers 5 mit einer neuen Reimfolge. Im Reimwechsel verdeutlicht Opitz die interne Gliederung des Oktetts in zwei Quartette und setzt ein Strukturelement ein, das den neulateinischen Versionen fehlt. Zusätzlich hat Opitz die Zweiteilung des Oktetts auch anaphorisch markiert: Während das erste Quartett durch vier IIst-Anaphern gekennzeichnet ist, wird das zweite jeweils durch ein Verb und das Personalpronomen \Ich/ eingeleitet.

Wie sehr Opitz auf die moderne Sonettform abhebt, zeigt auch sein eigenständiger Umgang mit der traditionellen Zweiteilung des Sonetts. Während Petrarca diese Grenze überspielt, indem er die lange Kette von Fragen auf die neunte Zeile ausdehnt, beendet Opitz sie schulmäßig mit dem achten Vers.

Daphnis 21 (1992), S. 27-43, könnten die auffälligen Abweichungen, die Opitz in seiner Version der Elegie I 18 des Properz vornimmt, den petrarkistischen Tenor der Nachdichtung stärken. Bezeichnenderweise integriert Opitz auch Petrarca und Laura in einen Katalog von Dichtern und ihren Geliebten, der antike und moderne Vorbilder integriert; vgl. M. O.: An Nüßlern. In: Gesammelte Werke. Bd. 2, 2 (Anm. 13), S. 646, V. 18.

15 So die These von Schulz-Buschhaus (Anm. 13), der freilich die lateinische Sonettistik nicht in Betracht zieht.

16 Das doppelte Paradoxon, welches »amor« [die 〉Liebe`] zum Liebesgott »Amor« personifiziert, schwächt Opitz zu zwei tautologischen Antithesen ab. Mein Befund widerspricht der These von Schulz-Buschhaus (Anm. 13), S. 80, der in der Opitzischen Version eine »lexikalische und semantische Emphatisierung « zu erkennen meint.

17 Die Regeln, die Opitz im Buch der Deutschen Poeterey für das Sonett aufstellt, lassen sich folgendermaßen zusammenfassen: 1 . Der Vers des Sonetts ist der Alexandriner oder der gemeine Vers, 2. Die Reimordnung in den Quartetten ist $a b b a a b b a$, männliche und weibliche Reime wechseln, 3. In den Terzetten sind zwei Reimpaare geboten. Doch handelt es sich keineswegs um starre Vorschriften, so daß der Verdacht von Gellinek (Anm. 13), S. 102, unangebracht ist, Opitz habe »den Oktettreim noch nicht als Formgesetz des Sonetts erkannt«. Maché (Anm. 4), S. 132, verweist zu Recht auf ähnliche Variationen der Sonettform in Italien und Frankreich. 
Deutlicher als das italienische Original betont die deutsche Nachdichtung auch die Eigenständigkeit des Sextetts, das sich syntaktisch abhebt. Besteht im Oktett jeder Vers aus einem einzigen Fragesatz, so sind es im Sextett jeweils zwei Verse, die zusammen einen Satz bilden; außerdem ist das Sextett anaphorisch als Einheit markiert, wobei die Anaphern des zweiten Quartetts invertiert werden. Die ungeraden Verse beginnen jeweils mit dem Personalpronomen IIch Anapher begrenzt, »Thue ichs nicht gern« und »Thue ich es gern« (V. 7-8).

Bei Opitz stehen sich also Oktett und Sextett formal wie gedanklich als klar getrennte Einheiten gegenüber: Das Oktett bemüht sich um eine kategoriale Erfassung des widersprüchlichen Zustandes der Liebe, wobei das erste Quartett allgemein gehalten, das zweite dagegen egozentrisch ist. Auf die Fragen des Oktetts antwortet das Sextett mit einer metaphorischen Illustration des paradoxen Zustands, der sich jeder kategorialen Einordnung entzieht.

Auch das Sextett hat Opitz eigenständig umgestaltet. So forciert er die petrarkistische Pluralität durch einen zusätzlichen Selbstvergleich des liebenden Ichs:

Ich wancke/ wie das Gras/ so von den kühlen Winden

$\mathrm{Vmb}$ Vesperzeit bald hin geneiget wirdt/ bald her.

Der Naturvergleich variiert den biblischen Topos vom `Gras` als Sinnbild der Vanitas. ${ }^{18}$ Die Bibel-Allusion wirkt wie eine moralische Selbstkritik und steckt auch den zweiten Vergleich an, den Opitz entsprechend ausmalt. Die Analogie der Vergleiche stärkt den Sinnzusammenhang des Sextetts, den Opitz metrisch wie syntaktisch hervorhebt: Während die Versenden im Oktett jeweils durch Fragezeichen deutlich markiert sind, bildet im Sextett jeweils ein Verspaar eine syntaktische Einheit. Die versübergreifende Syntax unterstützen Enjambements, welche die Versgrenzen von 9 und 10 wie von 11 und 12 relativieren und für einen lakonischen Sprechstil sorgen. Die Enjambements haben überdies eine mimetische Funktion: Sie bilden die Passivität des haltlosen lyrischen Ichs ab. Dieses ist als schwankes Gras dem Wind preisgegeben oder als Schiff ein Spiel der Wellen. Lautmalerisch werden die Fähmisse des lyrischen Ichs durch die alliterierenden $>\mathrm{W}$ ८-Laute illustriert. Besonders durch die doppelte sentenziöse Antimetabole des abschließenden Paarreims (syntaktischer Parallelismus mit lexikalischem Chiasmus) - mit identischen Binnenreimen - bringt Opitz Petrarcas Paradoxien und Antithesen auf suggestive Formeln. Auf diese Weise wird das unauflösliche petrarkistische Dilemma von Liebe und Liebesleid besser abgebildet, als es die Pointe eines lateinischen Epigramms vermag: Während bei Petrarca dieser Schluß unausges prochen bleibt, richtet Opitz das ganze Gedicht auf diese komplexe petrarkistische Doppelsentenz aus. Denn der gelungene Paarreim ist die Probe aufs Exempel für die Überlegenheit des Deutschen als Literatursprache.

18 Vgl. Vergänglichkeitspsalm 90 und weitere Belege in der Großen Konkordanz zur Lutherbibel. Stuttgart ${ }^{3} 1993$, s. v. »Gras«. Die implizite Transzendenz übersieht Maché (Anm. 4). 


\section{Wirkung des Mustersonetts: Parodien}

Mit seiner Version empfahl sich Opitz der späthumanistischen Gelehrtenrepublik als »deutscher Petrarca ${ }^{19}$ : Er erwies die Literaturfähigkeit des Deutschen, etablierte das Sonett als moderne Ausdrucksform und propagierte den Petrarkismus als zeitgemäßes Thema. Sein Francisci Petrarchae erlangte im 17. Jahrhundert die Kanonizität eines petrarkistischen Mustersonetts, ${ }^{20}$ das als Referenztext leicht wiedererkannt werden konnte. Denn es provozierte wie kein anderes Gedicht im 17. Jahrhundert zahlreiche parodistische Nachahmungen und Neuschöpfungen. Diese Nachahmungen beziehen sich auf Opitz, setzen sich aber auch allgemein mit der Sonettform, dem spezifischen Sprachstil und dem Liebesdiskurs des petrarkistischen Genres allgemein auseinander.

Die Opitz-Parodien ahmen den Prätext formal, strukturell und lexikalisch nach, verändern aber den Gehalt. Worin die intertextuelle Beziehung zwischen

19 Vgl. Gerhart Hoffmeister: Petrarkistische Lyrik. Stuttgart 1973, S. 66. Zu diesem Anspruch paßt Johann Rists Epicedium auf Martin Opitz:

Was rühm ich aber viel? Petrarcha soll es sagen

Der Florentiner Pracht, den Fama auff den Wagen

Der Ewigkeit gesetzt, den Opitz sehr geliebt

Und ihn in Teutsche Sprach zu bringen sich geübt.

Johan Rist: Lob- Trawr- vnd Klag-Gedicht/ Uber gar zu frühzeitiges/ jedoch seliges Absterben/ Des weiland Edlen/ Großachtbaren vnd Hochgelahrten Herren Martin Opitzen, Königlicher Majestät zu Pohlen wolbestalten Raths vnd Secretarij, Des allerberühmtesten Poeten zu vnseren Zeiten/ [...], Welcher am 6. Tage Septembris, des 1639. Jahres/ in der Königlichen Stadt Dantzig/ [...] in die Ewigkeit ist versetzet worden. Hamburg 1640, V. 153 160. Allerdings hatte Opitz lediglich ein zweites Sonett Petrarcas eingedeutscht: "Valle che de' lamenti miei se' piena « (Canz. 301); diese Übersetzung erschien in der Trostschrift an David Müller [1628]; M. O.: Gesammelte Werke (Anm. 13). Bd. 4: Die Werke von Ende 1626 bis 1630, Teil 1. Stuttgart 1988 (BLVS 312), S. 115f. Vgl. dazu die Bemerkungen von Wagner (Anm. 13), S. 276-279.

20 Vgl. Schulz-Buschhaus (Anm. 13), S. 79, der dem italienischen Original ২Kanonizität zuspricht. - In der Forschung blieb bislang unbeachtet, daß das Opitzische Mustersonett noch im 20. Jahrhundert Wirkung gezeitigt hat. So liefert es den Prätext für ein Exilgedicht Karl Wolfskehls, dessen beide Anfangsstrophen den Opitzischen Schluß variieren:

WILL NICHT was ich will. Will nicht was ich muss.

Die Trän ist trocken, verstaubt der Kuss.

Weiss nicht was ich weiss. Weiss nicht was ich soll,

Und schien doch aller Gnaden voll.

(K. W.: Gesammelte Werke. Bd. 1: Dramatische Dichtungen. Hg. von Margot Ruben und Claus Victor Bock. Hamburg 1960, S. 225, V. 1-4). Unverkennbar legt auch Friedrich Hollaender die Opitzische Antimetabole einer modernen Hysterica in den Mund:

Ich weiß nicht, was ich möchte,

Ich möcht nicht, was ich weiß,

Im Sommer ist mir frostig,

Im Winter ist mir heiß,

Mensch, bin ich hysterisch!

(F. H.: Die hysterische Ziege. In: F. H.: Lieder und Chansons für Blandine Ebinger. Mit Zeichnungen von Claus Arnold. Freiburg i. Br. 1957, S. 49-51, V. 9-13). 
Referenztext und Parodie besteht, ist freilich strittig. ${ }^{21}$ Historisch war der >Parodie--Begriff seit der Antike weit gefaßt und schloß neben Herabsetzungen im Stil der parodia iocosa ernste Posttexte vom Typ der parodia seria ein, umfaßte also gleichermaßen komische >Gegengesänge ‘ wie konkurrierende ernsthafte Nachahmungen. Diesen weiten Begriff haben Theodor Verweyen und Gunther Witting durch einen intensiven Parodie-Begriff ersetzt, der die >Parodie ‘ funktional auf die antithematische Komisierung eines Prätexts, auf die parodia iocosa, einengt und ihr eine "eigene Botschaft» abspricht. ${ }^{22}$ Die traditionelle parodia seria bezeichnen Verweyen und Witting dagegen als $>$ Kontrafaktur : Sie unterscheide sich "von der Parodie durch den Verzicht auf die Komisierung ihrer Vorlage(n) «. ${ }^{23}$ Der eingeschränkte >Parodie«-Begriff nach Verweyen/Witting hat nicht nur den Nachteil historischer Inadäquatheit, ${ }^{24}$ sondern engt auch die rezeptionsästhetische Vielfalt und Aussagekraft des Materials ein. Daher ist für unseren Überblick einer Wirkungsgeschichte von Opitz' petrarkistischem Mustersonett der traditionelle, weite `Parodie〈-Begriff hinreichend trennscharf und sinnvoll.

Die bislang nur mangelhaft erforschte Wirkungsgeschichte von Opitzens Petrarca-Übersetzung stellt somit ein wichtiges Dokument für den Petrarkismus im deutschen Barock dar. ${ }^{25}$ Anhand der Variationen und Parodien auf Opitzens Sonett Auß dem Italienischen Petrarchae läßt sich in einer Fallstudie der Wandel des petrarkistischen Diskurses im 17. Jahrhundert nachzeichnen. In intertextuellen Analysen werden die verschiedenen Formen des Zitierens, Thematisierens und Imitierens (Peter Stocker) erörtert, um festzustellen, inwieweit die Parodien das Opitzische Muster nachahmen, kritisieren oder überbieten

21 Für die `Literatur zweiten Grades` liegen zahlreiche terminologische Abgrenzungen vor, die

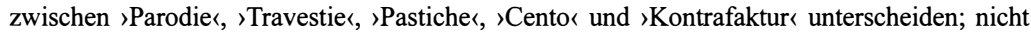
durchsetzen konnte sich das kommunikationstheoretische Modell von Hans Kuhn: Was parodiert die Parodie? In: Neue Rundschau 1974, S. 600-618. Ebenso folgenlos blieb der Ansatz des Praktikers Robert Neumann: Zur Ästhetik der Parodie. In: R. N.: Die Parodien. München, Wien und Basel 1962, S. 553-563, vom Referenztext auszugehen (»nur das Geformte ist parodierbar«, ebd., S. 556).

22 Vgl. Theodor Verweyen und Gunther Witting: Parodie. In: Reallexikon der deutschen Literaturwissenschaft. Bd. 3: P-Z. Berlin und New York 2003, S. 23-27. Hier wird die definitorische Abgrenzung des engen >Parodie`-Begriffs zur `Kontrafaktur` dekretiert, die Verweyen und Witting in früheren Studien noch als pragmatische Entscheidung relativierten; vgl. etwa das Nachwort zu: Deutsche Lyrik-Parodien aus drei Jahrhunderten. Hg. von Th. V. und G. W. Stuttgart 1984, S. 299-321, bes. 306.

23 Theodor Verweyen und Gunther Witting: Kontrafaktur. In: Reallexikon der deutschen Literaturwissenschaft. Bd. 2: H-O. Berlin und New York 2000, S. 337-340, hier 337.

24 Den Wandel des musikalischen Begriffs zu einem literarischen Terminus erhellt Hermann Koller: Die Parodie. In: Glotta. Zeitschrift für griechische und lateinische Sprache 35 (1956), S. 17-32.

25 Die Opitz-Parodien sind meines Wissens bislang noch nicht umfassend gesammelt worden. Deutsche Parodien auf Opitz' Petrarca-Version finden sich in der Textsammlung von Luzius Keller (Anm. 6) (Fleming) sowie in den Anthologien von Jörg-Ulrich Fechner (Anm. 9) (Tilenus, Fleming, Logau) sowie von Ulrich Maché und Volker Meid (Hgg.): Gedichte des Barock. Stuttgart 1980 (Homburg, Schwarz, Schirmer). 
- >Teilparodien bleiben außer Acht. ${ }^{26}$ Ziel ist es, den barocken Petrarkismus in einer ১dichten Beschreibung zu differenzieren und zu periodisieren.

Das Korpus der Nachgeschichte bilden sechs Parodien, die sich zeitlich in drei Gruppen gliedern lassen. Die Parodien von Ernst Christoph Homburg (1605-1681), Paul Fleming (1609-1640) und Sybilla Schwarz (1621-1638), die allesamt um 1635, also noch zu Lebzeiten von Martin Opitz entstanden, bilden die erste Gruppe. Eine zweite Gruppe, die Bearbeitungen von David Schirmer (1623-1687) und Friedrich von Logau (1605-1655), entstammt der Mitte des 17. Jahrhunderts, während die Parodie von Daniel Casper von Lohenstein aus dem letzten Drittel des Jahrhunderts einer dritten Phase zuzuordnen ist.

\subsection{Parodien zu Lebzeiten von Martin Opitz}

Die Parodien von Paul Fleming, Sybilla Schwarz und Ernst Christoph Homburg bezeugen - bei aller Verschiedenheit - einerseits die literarische Autorität von Martin Opitz, andererseits eine gewisse Distanzierung von seiner späthumanistischen Ausrichtung. Die aemulatio der Verfasser mit Opitz zeigt sich formal wie inhaltlich. So wird die unorthodoxe Sonettform abgewandelt, aber auch sein klassizistisch gedämpfter Petrarkismus kritisiert. Vor allem aber führt man gegen den Opitzischen Sonettpetrarkismus einen moralischen Antipetrarkismus ins Feld, der freilich ganz uneinheitlich ist. Seine Spielarten reichen von formästhetischer Überbietung bis zu moralischer Ablehnung. Da bislang lediglich Flemings Variation genauer untersucht wurde, blieb auch die Vielfalt der frühen petrarkistischen Positionen unberücksichtigt.

\subsubsection{Paul Fleming: Wie? Ist die Liebe nichts? (1635)}

Bereits die frühen neulateinischen Kuß-Gedichte Paul Flemings (Rubella, 1631) antizipieren mit ihren Antithesen und Paronomasien den Petrarkismus der deut-

26 Die intertextuellen Kategorien (Zitieren, Thematisieren, Imitieren) von Peter Stocker: Theorie der intertextuellen Lektüre. Paderborn 1998, scheinen mir zur Analyse von Parodien geeignet. - Zum Verhältnis von imitatio und aemulatio, den produktionsäthetischen Leitkategorien in den rhetorisch dominierten Poetiken des 17. Jahrhunderts, vgl. HansJoachim Lange: Aemulatio veterum sive de optimo genere dicendi. Die Entstehung des Barockstils im 16. Jahrhundert durch eine Geschmacksverschiebung in Richtung der Stile des manieristischen Typus. Bern und Frankfurt 1974, Wilhelm Kühlmann: Petrarkistische Ovidimitatio (Anm. 14), und Barbara Bauer: Aemulatio. In: Historisches Wörterbuch der Rhetorik. Hg. von Gert Ueding. Bd. 1. Tübingen 1992, Sp. 141-187. - Unberücksichtigt bleiben >Teilparodien`, die nur bestimmte Aspekte des Prätexts nachahmen (Form, Fragenkatalog, erotische Dilemmata, Bildlichkeit), wie z. B. Johann Rist: Sie rühmet ihre Beständigkeit. In: Gedichte des Barock (Anm. 25), S. 71 f., V. 3 f. (Schiffsvergleich), Sybilla Schwarz: »Ist Lieb ein Feur«. In: Ebd., S. 181 (Fragenkatalog, Feuer-Eis-Opposition), und David Schirmer: An sich selbst (»Du/ warümb bistu blaß?«; vgl. den Text in Anm. 39) (Fragenkatalog, Feuer-Kälte-Opposition, Schlußpointe). 
schen Gedichte, zu denen Martin Opitz Fleming persönlich ermuntert hatte. Muster und Liebesauffassung des Petrarkismus machte sich Fleming mittels Nachahmung, Übersetzung und Paraphrase zu eigen. ${ }^{27}$ Wie virtuos der fünfundzwanzigjährige Fleming die Register des Petrarkismus beherrschte, zeigt seine Parodie auf das petrarkistische Mustersonett des Martin Opitz. Sie findet sich in dem Gedichte Auff des [...] Reineri Brockmans [...] Hochzeit, einem prosimetrischen Hochzeitsgedicht aus dem Jahre 1635:

Wie? ist die Liebe nichts? was liebt man denn im Lieben? was aber? Alles? Nein. wer ist vergnügt mit ihr? nicht Wasser. sie erglüt die Hertzen für und für. Auch Feuer nicht. Warumb? Was ist für Flammen blieben?

Was denn? Glut? aber sagt/ woher kömpt ihr betrüben? denn Böse? mich dünckts nicht. nichts solches macht Begier. Den Leben? nein. wer liebt/ der stirbt ab seiner Zier/ und wird bey Leben schon den Todten zugeschrieben.

So wird sie todt denn seyn? nichts minder/ als diß eben. Was tod ist/ das bleibt todt. aus liebe kompt das Leben. Ich weiß nicht/ wer mir sagt/ was? wie? wo? oder wenn? Ist nun die Liebe nicht Nichts? Alles? Wasser? Feuer? Gut? Böse? Leben? Todt? Euch frag' ich newe Freyer/ Sagt ihr mirs/ wenn ihrs wisst: was ist die Liebe denn? ${ }^{28}$

Der Eingangsvers gibt das Incipit von Opitzens »Ist Liebe lauter nichts« wörtlich wieder. Damit ist der intertextuelle Bezug hinlänglich markiert, zumal der Eingang von Vers 11 noch den Anfang der Opitzischen Schlußpointe zitiert (»Ich weiß nicht«). Indem Fleming das Opitzische Incipit mit einem unvermittelt vorgeschalteten »Wie« in Frage stellt, verdeutlicht er nicht nur die Markierung, sondern betont auch die Dialogizität seines eigenen Sonetts und weist es als parodischen Gegenentwurf aus.

$\mathrm{Da} ß$ Fleming nicht Opitz allein, sondern das petrarkistische System insgesamt im Visier hat, zeigt die Sonettform. Denn Fleming vereinheitlicht die Reimordnung im regelpoetischen Sinne: er wechselt den Reim nicht in den Quartetten, sondern erst in den Terzetten, die, wie es Opitz in der Deutschen Poeterey empfiehlt, mit einem Reimpaar beginnen. Auch ohne Enjambements wirkt Flemings Sonett dialogisch-lebendig. Dieser Eindruck resultiert aus einer Fülle unverbundener, kleiner, oft elliptischer syntaktischer Einheiten. Die aussparende Percursio ist zusätzlich durch die Fiktion eines Zwiegesprächs dynamisiert. Fleming veräußerlicht so das Selbstgespräch, in dem Opitz ein lyrisches Ich die Wirkungen der Liebe erörtern läßt, zu einem zweistimmigen Frage-Antwort-Spiel. Da die Fragen über das Wesen der Liebe stark verkürzt

27 In den amourösen Oden und Sonetten kombiniert Fleming, wie Hans Pyritz: Paul Flemings Liebeslyrik. Zur Geschichte des Petrarkismus. Göttingen 1963, nachwies, neulateinische und petrarkistische Traditionen. Vgl. auch Zon (Anm. 13), S. 506-511.

28 Paul Fleming: »Wie? ist die Liebe nichts? «. In: P. F.: Auff des [...] Reineri Brockmans [...] Hochzeit. Reval (Chr. Reusner) 1635; wieder in P. F.: Deutsche Gedichte. Hg. von Volker Meid. Stuttgart 1986, S. 10-38, hier 36. Emendiert ist der Schluß von Vers 7: »Zier/« statt »Zier." 
und - von acht auf siebenundzwanzig - mehr als verdreifacht sind ${ }^{29}$ wirkt das Tempo überhitzt, der Sprachstil eher familiär-kolloquial und der dialektische Charakter übertrieben. Die dynamisierende Übertreibung von Stilmerkmalen des Prätexts - etwa fünf Interrogativpronomina in Vers 11 - dient der parodistischen Überbietung des petrarkistischen Musters. Das Tod-Leben-Thema findet sich nicht bei Opitz, wohl aber bei Petrarca selbst, der in Vers 7 die Liebe als "viva morte" apostrophiert. Fleming greift somit über den Vermittler Opitz direkt auf Petrarca zurück, zweifelt aber Petrarcas Paradoxon an. Denn die Alternative der fragenden Stimme, ob Liebe `Leben oder Tod bedeute, wird von der antwortenden Stimme doppelt verneint.

Der Anfang von Vers 11 - »Ich weiß nicht, wer mir sagt, was, wie, wo oder wenn?« - alludiert die Schluß-Sentenz der Opitzischen Version, bevor er noch einmal in asyndetischer Häufung alle vorgängigen Antitheta resümiert, mit denen die Liebe gleichgesetzt wurde, um dann in einer witzigen Apostrophe das Brautpaar zu bitten, das Liebesdilemma aufzulösen. Der Bedingungssatz (»wenn ihrs wißt«) ironisiert freilich die angebliche Kompetenz der Befragten. Doch hebt die ironische Schlußwendung die antipetrarkistische Tendenz des Sonetts nicht auf. Denn Flemings Opitz-Parodie führt in der hyperbolischen Intensivierung aller petrarkistischen Register den antithetischen Automatismus des Petrarkismus ad absurdum: Die Amplitude der widersprüchlichen Liebesgefühle wird so sehr dramatisiert, daß sie sich jeder Systematisierung entziehen.

$\mathrm{Da}$ Fleming mit seinem Sonett Opitz parodierte, war den Zeitgenossen bewußt. Denn nach Vortrag des Gedichts erkennt Flemings Dichterfreund Olearius ausdrücklich an: »Ein gut Stücke [...] welches wol weiset/ bey was vor einem Meister er in die Schule gangen «. ${ }^{30}$ Doch läßt dieses Lob sich auch im Sinne einer aemulatio als eine Überwindung des Meisters Opitz und seines petrarkistischen Systems lesen.

\subsubsection{Ernst Christoph Homburg: [XXVII.] Epigramma (1642)}

Fast gleichzeitig mit Fleming hat Ernst Christoph Homburg das petrarkistische Mustersonett des Martin Opitz parodiert. Homburg deutet mit dem Titel »Epigramm« die Tendenz seiner Bearbeitung an. Sein »Epigramm« besteht aus drei vierzeiligen Alexandriner-Strophen und einem Couplet. Es gemahnt an den Typ des englischen Sonetts und ähnelt der epigrammatischen Form, die Martin Opitz seinem Mustersonett gegeben hat:

29 In den postumen Teutschen Poemata. ${ }^{\mathrm{r}}$ (Lübeck [1642]) Hildesheim 1969, S. 574, weist das Gedicht 26 Fragezeichen, in der in editionsphilologischer Hinsicht ebenso problematischen Ausgabe J. M. Lappenbergs (P. F.: Deutsche Gedichte. 2 Bde. Stuttgart 1865, S. 92f.) nur 16 Fragezeichen auf. In neueren Drucken, in denen Orthographie und Zeichensetzung modernisiert sind (z. B. Fechner [Anm. 9], S. 71), ist die Zahl der Fragezeichen ebenfalls meist deutlich reduziert.

30 Paul Fleming: Deutsche Gedichte (Anm. 28), S. 36. 


\section{[XXVII.] Epigramma}

ISt Liebe Zuckersüs/ wie daß sie bitter schmecket?

Ist Liebe bitter Gall/ wie daß sie Lust erwecket?

Ist Liebe lauter Fewr/ wie daß sie Thränen bringt?

Ist Liebe lauter Flut/ daß ihre Glut dann dringt

$\mathrm{Zu}$ innerst in das Hertz? Ist Liebe was zu nennen?

Wer ist dann der/ und die/ so Liebe recht mag kennen?

Ist Liebe lauter nichts/ wie kann und mag es seyn/

$\mathrm{Da} ß$ sie bringt dir und mir wol tausend Höllen-Pein?

Ist Liebe Menschen-Werck/ wie daß sie Götter drenget?

Ist Liebe GötterThun/ wie daß sie sich vermenget

Mit dem/ was menschlich ist? Ist Liebe heilsam-gut/

Wie daß sie dann so gar verformet Hertz und Mut?

Drumb wer will witzig seyn/ und Fillis ihm erjagen/

Der wird/ was Lieben sey/ mir kürtzlich Antwort sagen. ${ }^{31}$

Wie Opitz verwendet Homburg sieben Reime - ungewöhnlich viele Reime für ein Sonett -, doch ordnet er sie wie Distichen ausschließlich paarweise an, wobei er das Reimgeschlecht alternieren läßt.

Das Incipit von Opitz findet sich - in die Mitte des Gedichts versetzt - wörtlich zitiert: »Ist Liebe lauter nichts« (V. 7). Präludiert wird das prägnante Zitat durch zwei Paraphrasen (»Ist Liebe lauter Fewr« [V. 3] und »Ist Liebe lauter Flut« [V. 4]). Auch syntaktisch folgt Homburg dem Prätext, indem er zwei charakteristische Satzgebilde von Opitzens Petrarca-Sonett aufgreift und intensiviert: die anaphorische »Ist Liebe«-Reihe uneingeleiteter Bedingungssätze und die durch das Interrogativpronomen »wie daß« eingeleiteten Fragesätze. ${ }^{32}$ Doch anders als Opitz bindet Homburg die Syntax nicht an die Sonettstruktur: Während Opitz seine Kombination von Bedingungs- und Fragesatz auf das Oktett beschränkt, dehnt Homburg sie auf sechs der sieben Reimpaare aus. Sein letztes Reimpaar wirkt durch das einleitende $»$ Drumb « wie eine logische Konklusion aus den insgesamt neun Fragen. Damit spitzt Homburg seine Parodie auf die Schlußpointe zu und rechtfertigt so den Gattungsnamen »Epigramm« im Titel.

Die Bedeutung der Pointe erschließt sich erst vor dem Hintergrund der als Prämissen fungierenden Fragesätze. Homburg intensiviert die Antithesen pleonastisch, vor allem in Form von Hyperbeln (»lauter«, »tausend«), konkretisierenden Metaphern (»zuckersüß«, »bitter Gall«, »innerst in das Hertz«) und Zwillingsformeln (»der und die«, »kan und mag«, »heilsam-gut«, »Hertz und Mut«). Dank pleonastischer Intensivierung sowie stärkerer Figurierung - mehr Anaphern und Parallelismen - und syntaktischer Vereinheitlichung ist Hom-

31 Ernst Christoph Homburg: [XXVII.] Epigramma. In: E. Chr. H.: Schimpff- vnd Ernsthaffte Clio Erster Theil. Zum andern mal vmb die Helffte vermehret [...] / verbessert. Jena (B. Lobenstein für Z. Hertel) 1642, Bl. V 4vf. Wieder in: Maché und Meid (Anm. 25), S. 97.

32 Homburg verwendet neunmal die Wendung »Ist Liebe«, davon siebenmal am Versanfang, zweimal zur Fortführung nach der Alexandrinerzäsur; die »wie daß«-Formel verwendet Homburg sechsmal, zwei weitere Male variiert (durch Sperrung: »wie [...] daß« [V. 7f.] bzw. Ersparung des >wie` [V. 4]). 
burgs Parodie viel emphatischer als der Prätext. Daß die Emphase der Passion eines liebenden Ichs entspricht, bekundet das mittlere Verspaar, das syntaktisch eine Einheit bildet und neben der zentralen Position noch durch das Zitat des Opitz-Incipits hervorgehoben ist: Es ist das einzige Mal im Vorbereitungsteil des Epigramms, daß sich das lyrische Ich nennt:

Ist Liebe lauter nichts/ wie kan und mag es seyn/

$\mathrm{Da}$ sie bringt dir und mir wol tausend Höllen-Pein?

Das lyrische Ich drückt seine lebhafte Teilnahme in einem doppelten Dativ ethicus aus: »dir und mir«. Auch wenn im 〉Du eine Geliebte angesprochen sein könnte, legt die Schlußpointe eine Empathie-Formel, eine Anrede an den mitleidigen Leser nahe. Denn hier wird deutlich, daß das lyrische Ich klagt, weil es von der Geliebten, die den antiken Schäfernamen »Fillis « trägt, ${ }^{33}$ nicht erhört wird. Deswegen bittet das lyrische Ich resigniert die switzigen die Gunst der spröden Schäferin erlangen, ihn kurz über die Liebe aufzuklären. Doch zeigt die venatorische Metapher vom serjagen` der Liebesbeute, daß das lyrische Ich vor einer galanten Liebesauffassung kapituliert, die sich von seiner Liebesleidenschaft diametral unterscheidet.

Die petrarkistische, mit »Hertz« und »Pein« untrennbar verbundene Liebesauffassung, wie sie das lyrische Ich in den ersten sechs Reimpaaren darlegt und mit dem apostrophierten Leser teilt, wirkt antiquiert in einer Zeit, in der Rivalen sich mit galantem $>$ Witz ihre Liebesbeute erjagen. Homburg erweist mit seiner Parodie, daß das von Opitz etablierte System des Petrarkismus nur in der Literatur Chancen hat, aber nicht im Leben: Er würdigt und kritisiert somit in sentimalischer Brechung die hohe, aber überholte Liebe, an deren Stelle die galant-witzige Verführung getreten ist.

\subsubsection{Sybille Schwarz: Ist Lieben keusch? (vor 1638)}

Die frühverstorbene Dichterin Sybille Schwarz - sie starb noch nicht 18jährig 1638 in ihrer Heimatstadt Greifswald - hat wohl kurz nach Fleming und Homburg die Opitzische Petrarca-Version zum Vorwurf eines eigenen Sonetts genommen:

Ist Lieben keusch? wo kompt denn Ehbruch her?

Ist Lieben guht, nichts böses drinn zu finden,

wie kan sein Feur dan so gahr viel entzünden?

Ist Lieben Lust, wer bringt dan das Beschwär?

Wer Lieben liebt, fährt auff der Wollust Meer, und lässet sich ins Todes Netze binden, das nicht zerreist, er lebet nuhr den Sünden, liebt Eitelkeit, und ist der Tugend leer.

Das ewig lebt, dem stirbt er gäntzlich ab, sieht seine Noht erst, wan er siht sein Grab.

33 Seit Vergils Georgica bezeichnet Phyllis topisch ein liebenswertes Bauernmädchen. 


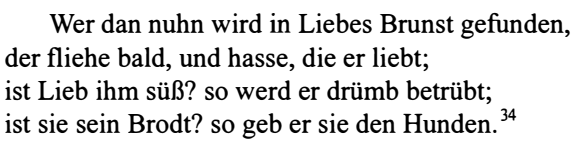

Schwarz hält sich genauer als Opitz selbst an die Sonett-Regeln, die dieser im Buch von der Deutschen Poeterey aufgestellt hat. Ihre beiden Quartette weisen ein und denselben Reim auf, ihre Terzette die von Opitz bevorzugten Paarreime. Da Schwarz die vier Schlußverse wie eine Quartettstrophe anordnet, ist das Reimpaar der Verse 9 und 10 als Sentenz wie eine eigene Strophe hervorgehoben und korrespondiert so mit dem Opitzischen Schlußcouplet. Auch sonst variiert Sybille Schwarz die Metrik. Sie verwendet - übrigens als einzige Parodie - keine Alexandriner, sondern sogenannte `Gemeine Verse $\iota, d$. h. jambische Fünfheber. Den svers commun hatte Opitz in der Poeterey zwar für zulässig erklärt, doch dominierte in der Dichtung des 17. Jahrhunderts eindeutig das Alexandrinersonett. Die metrische Abweichung kompensiert Schwarz durch palintextuellen Reim, indem sie Opitzens unreine weiblichen $i / \ddot{u}$-Reime (»entzündet« / »empfindet« bzw. "führ« / »mir«) nachahmt und sogar die Reimwörter »finden « / »entzünden« zitiert. Zusätzlich markiert die erste Strophe die Bezugnahme auf Opitzens petrarkistisches Sonett stilistisch und syntaktisch, durch das anaphorische >ist und vier Fragesätze.

Inhaltlich kehrt Schwarz das petrarkistische Liebesgeständnis im Sinne einer moralisatio um. Diese Tendenz zeigt bereits der erste Vers, der die ethische Qualität der Liebe und den kausalen Zusammenhang zwischen \Liebe` und Sünde ( (Ehebruch $८$ ) rhetorisch in Frage stellt. So wird das Liebesthema nicht mehr in aporetischen Dilemmata umschrieben, sondern explizit genannt. ${ }^{35}$ Mit der moralisatio geht auch eine Entindividualisierung einher. Indem Schwarz nur die dritte Person Singular verwendet, wahrt sie Distanz und meidet die subjektive Perspektive eines lyrischen Ichs. Besonders markant wird diese Differenz in der Bildlichkeit. So wird die Not von Petrarcas liebendem Ich, die im Selbstvergleich mit einem »Schiff« zum Ausdruck kommt, »daß in dem wilden Meer | Von Wellen umbgejagt" wird, von Schwarz in einer Figura etymologica als sündhaft diskreditiert: »Wer Lieben liebt, fährt auff der Wollust Meer« (V. 5). Die schärfste Diskrepanz zur Vorlage gewinnt die moralisatio in dem Verspaar 9/10, welches das Sextett einleitet. Anders als in der kunstvollen Antimetabole des Prätextes wird die Verfehlung weltlicher Liebe sub specie aeternitatis in einem Chiasmus (»ewig lebt« - »stirbt [...] gäntzlich«) und einem Parallelismus (»sieht seine Noht« - »sieht sein Grab«) verdeutlicht. Die Schlußstrophe nach der Sentenz mahnt den Liebenden zur Umkehr. In den beiden abschließenden Versen wird der petrarkistischen Liebe eine drastische

34 Sibillen Schwarzin [...] Ander Teil Deutscher Poëtischer Gedichten [...] Danzig 1650 [Repr. 1980], Bl. P 1 ${ }^{v}$. Wieder in Helmut W. Ziefle: Sybille Schwarz. Leben und Werk. Bonn 1975, S. 184.

35 Während Opitz das Nomen ıLiebe` und das Verbum slieben` jeweils nur ein einziges Mal gebraucht, verwendet Schwarz die beiden Begriffe insgesamt neunmal. 
Absage erteilt. Ihr syntaktischer Parallelismus alludiert die sentenziöse Antimetabole des Martin Opitz. Die ersten Vershälften greifen die >Ist<-Fragen nach dem Wesen der Liebe auf, um sie nach der Zäsur mit antithetischen >So〈Folgerungen $\mathrm{zu}$ bescheiden: die weltliche Liebe - so der krude antipetrarkistische Widerruf - wird den Hunden zum Fraß vorgeworfen.

\subsection{Parodien um die Jahrhundertmitte}

Die Dichtergeneration, die um 1650 das literarische Feld beherrscht, unterzieht die Petrarca-Version des Martin Opitz erneut einer parodistischen Revision. In diesen Parodien kommen zwei unterschiedliche ästhetische Orientierungen zum Ausdruck: zum einen eine christlich-moralische Tendenz, die - wie zuvor Sybille Schwarz - das Liebesthema transzendiert und verernstigt, zum andern eine höfisch-internationale Strömung, die den Petrarkismus spielerisch weitertreibt.

\subsubsection{Friedrich von Logau: In Person eines Wittibers (1654)}

Friedrich von Logau repräsentiert eine Dichtergeneration, die sich vom lateinischen Späthumanismus löst und moralischen wie kulturpatriotischen Anliegen zuwendet. Von der selbstbewußten aemulatio dieser Generation mit Martin Opitz zeugt Logaus Opitz-Variation aus dem Jahre 1654:

In Person eines Wittibers
BRingt lieben etwa Lust/ bringt Lust von Liebe sagen/
Bringt beydes dennoch mir nichts als nur Bittrigkeit/
Was andren Hertzens-Wonn/ ist mir nur Hertzens-Leid/
Dann meine Lieb ist längst zu Grabe weg getragen;
Wiewol/ wer recht geliebt/ pflegt nichts darnach zu fragen/
Er liebet fort vnd fort/ vnd hat erst auß geliebt
Wann jhm sein Ende selbst deß liebens Ende gibt;
Die Liebe war nicht starck die sich verzehrt von Tagen.
Ich liebe weil ich bin! die nicht mehr ist zu lieben
Erfodert jhre Trew/jhr Werth ist ewig werth
Daß mehr als nur von jhr/ mein Mund kein Wort begehrt/
Mein Sinn sonst keine Lust; hieran wil ich mich üben!
Geht dieses lieben gleich bey andren bitter ein/
Soll mir um Liebe doch/ Lieb auch das bittre seyn. ${ }^{36}$

Logau hat In Person eines Wittibers, sein einziges Sonett, vor allem stilistischmetrisch als Opitz-Parodie markiert. Zwar zitiert auch das Incipit »Bringt lieben

36 Salomon von Golaw [Ps. Friedrich von Logau]: In Person eines Wittibers. In: F.v. L.: Sinn-Gedichte. ${ }^{\mathrm{r}}$ (Breslau [G. Gründer für C. Kloßmann] [1654]) Hildesheim und New York 1972, S. 135; Wieder in: F. v. L.: Sämtliche Sinngedichte. Hg. von Gustav Eitner. Tübingen 1872 (BLVS 113), S. 343, und Fechner (Anm. 9), S. 74. 
etwa Lust « unverkennbar das moralische Dilemma, das Opitz antithetisch faßt: »Ist sie [scil. die Liebe] recht und gut/ wie bringt sie böse Lust?«. Das »etwa« macht deutlich, daß sich das Gedicht auf eine vorgängige Position bezieht. Noch deutlicher sind die rhetorisch-syntaktischen Entsprechungen zu Beginn des Gedichts. Indem Logau die Konjunktionen des Konditionalsatzes durch Anfangsstellung des Verbs erspart und das Eingangsverb zweimal wiederholt, imitiert er die anaphorische Manier des Prätexts. Eine entsprechend allusive Funktion haben die einleitenden Antithesen ebenso wie der syntaktische Parallelismus bei lexikalischem Chiasmus im Eingangsvers - unverkennbar eine Reminiszenz der charakteristischen Antimetabole von Opitz.

Äquivalenz und Differenz prägen auch das metrische Verhältnis von Muster und Parodie. Wie Opitz folgt Logau dem englischen Sonett-Typ, doch behält er im Unterschied zu Opitz den umarmenden Reim im Oktett bei, lediglich der eingeschlossene Reim wechselt. Das Sextett ist übereinstimmend mit Opitz durch auffällige Anfangsstellung des »Ich" gekennzeichnet. So sehr sich Logaus Variation des englischen Sonett-Typs an Opitz orientiert, so sehr bekundet Logau seine poetische Selbständigkeit: Er übernimmt kein einziges Reimwort.

Thematisch folgt Logau dem petrarkistischen Liebesgedicht, ${ }^{37}$ macht jedoch aus einem Liebesbekenntnis ein Rollengedicht ehelicher Treue: Logau widmet die petrarkistische Hingabe in eine Liebe über den Tod hinaus um. Paronomasien und Polyptota, variierende Wiederholungsfiguren (»liebet $[\ldots]$ auß geliebt«, »fort vnd fort«, »ihr Werth ist ewig werth«, »sein Ende $[. .$.$] deß$ liebens Ende«, »um Liebe [...] Lieb«) und Distinktionen (»Hertzens-Wonn« vs. »Hertzens-Leid«) markieren die thematische Differenz zur petrarkistischen Vorlage. Aus der Haltlosigkeit des lyrischen Ichs wird in Logaus Rollengedicht ein selbstbestimmtes Lebenskonzept, das nicht nach den »andren « fragt: Der treue Witwer, der nur einer einzigen Liebe lebt, faßt dies wie Opitz in einer paradoxen Reimpaar-Pointe zusammen, die das Sonett beschließt:

Geht dieses lieben gleich bey andren bitter ein/

Soll mir um Liebe doch/ Lieb auch das bittre seyn.

Indem das Rollen-Ich an der unzeitgemäßen Umwidmung einer das Grab überdauernden Liebe festhält, wird dem Opitzischen Petrarkismus ein moralisch überlegener, metaphysischer Petrarkismus entgegengehalten.

\subsubsection{David Schirmer: Uber seine Träume (1657)}

Noch deutlicher wird die Distanz zum späthumanistischen Petrarkismus Opitzischer Prägung in David Schirmers Variation. Schirmer, seit 1650 Hofdichter in Dresden, gehört der Nachkriegsgeneration der Barockdichter an, die sich galanten und manieristischen Einflüssen öffnen. Die Parodie des Opitzischen Mustersonetts findet sich in seinen petrarkistischen Marnia-Sonetten. Sie zeich-

37 Dreizehnmal kommt das Wort `Liebe` als Nomen, Verb oder Adjektiv vor. 
nen sich durch ein spielerisches Verhältnis zur Tradition aus und setzen Zitat, Imitation und Parodie als wichtige Stilelemente ein:

\section{Uber seine Träume}

SInd Träume lauter nichts/ wie daß sie mich bewegen? sind sie denn Freud und Lust/ wie daß ich traurig bin? sind sie vol Lieblichkeit/ wie daß mein toder Sinn sich muß/ O Marnie/ zu deinen Füssen legen.

Ich sahe heint zu Nacht dich deiner Liebe pflegen.

Du warst es ja gewiß/ O schöne Halb-Göttin.

Ein nacket Nymphen-Bild lief zu den Schwanen hin/ zun Schwanen/ die im Thal stets ihre Lieder hegen/

Und küsset eines Mund. Ich fühlte Süssigkeit.

Die Liebe stieß alsbald nach meinem krancken Hertzen.

Drauf ließ ich meinen Schlaff. Nichts blieb als tausend Schmertzen/ die ich noch klagen muß bey später Abends-Zeit.

Sie sind nun was sie sind/ so gläub in vollen Sorgen/ im Traume-Nebel liegt die Warheit doch verborgen. ${ }^{38}$

Schirmer verwendet das übliche Alexandriner-Sonett, allerdings mit gleichem Reim in den Quartetten ( $a b b a a b b a$ ). Dagegen stimmt die Reimordnung der Terzette ( $c d d c e e)$ mit der Opitzischen Petrarca-Version überein, so daß ein Reimpaar das Sonett beschließt. Zudem folgt Schirmer dem Prätext im Wechsel der Versschlüsse.

Schirmer ersetzt das Substantiv 〉Liebe` durch 〉Träume`, erinnert aber durch seine >wie daß<-Fragen und syntaktischen Konstruktionen an die Vorlage. Außerdem ist die Sprechsituation entscheidend verändert: Schirmer macht aus dem petrarkischen Soliloquium eines liebeskranken Ichs die persuasive Ansprache eines liebenden Dichters an die Geliebte. ${ }^{39}$ Der Fragenkatalog des lyrischen

38 David Schirmer: Uber seine Träume. In: D. S.: Poetische Rosen-Gebüsche [I 3, 5]. Dresden (M. Berg für A. Löffler) 1657, S. 167 (Reprint. Hg. von Anthony J. Harper. Tübingen 2003 [Deutsche Neudrucke. R. Barock. 42/2]); wieder in Maché und Meid (Anm. 25), S. 222. Zu den Petrarca-Bezügen Schirmers vgl. Hugo Souvageol: Petrarca in der deutschen Lyrik des 17. Jahrhunderts. Ein Beitrag zur Geschichte der italienischen Literatur in Deutschland. Ansbach 1911, S. 43-51, und Anthony J. Harper: David Schirmer - a Poet of the German Baroque. An examination of Schirmer's lyric poetry and its relationship to the literature of the time. Stuttgart 1977, S. 140-145.

39 Wie bewußt David Schirmer die monologische Sprechsituation in eine dialogische verwandelt, zeigt seine andere Parodie auf das Opitzische Petrarca-Sonett, das als Selbstgespräch gestaltet ist: D. S.: An sich Selbst. In: Poetische Rosen-Gebüsche [I 3, 53] (Anm. 38), S. $217 f$.

An sich Selbst

DU/ warümb bistu blaß? Das Blut entgehet mir/ und nimt die Zuflucht hin zu meinem krancken Hertzen.

Warümb verlescht dein Licht? Ich fühle tausend Schmertzen.

Warümb denn weinestu? Die Thränen sollen hier

Der Liebe Gluth und Brunst ertödten für und für/ denn meine Flamme brennt in ihren vollen Kertzen. Was hilfft das seufftzen dich? Die Hitze zu verschertzen/ und mit gekühlter Luft zudämpfen die Begier. 
Ichs nach dem Wesen der Träume sind nur die scheinbare spropositior. Die anschließende rargumentatio<, mit der ein Wechsel ins Präteritum einhergeht, erstreckt sich als kleine Erzählung bis ins zweite Terzett hinein. Der Verfasser berichtet von einem nächtlichen Traum, in dem er seine Geliebte Marnia die Schwäne küssen sah. Da Schwäne traditionell das Wappentier der Dichter sind, ${ }^{40}$ hat die Traumerzählung weniger reflexiven, als vielmehr appellativen Charakter. Dies verdeutlicht die epigrammatische Schlußpointe, welche der Geliebten Marnia die Wahrheit des Traums bekräftigt (»so gläub«). Mit der tautologischen Eingangsfloskel (»Sie [scil. die Träume] sind nun was sie sind» [V. 13]), deren Binnenreim die Opitzische Pointe alludiert, wechseln Tempus und Sprechstil. Der Sprecher relativiert nachträglich den rhetorischen Vorwand seiner Propositio. Er gibt freimütig zu, weniger an der Natur der Träume interessiert zu sein, als an ihrer Realisation, indem er der Geliebten Marnia nahelegt, den Dichterschwan wie im Traum zu küssen. Es ist bemerkenswert, daß in der galanten Lyrik der sogenannten zweiten Schlesischen Schule das Sonett häufig wieder die epigrammatische Struktur erhält, die ihm Opitz ein halbes Jahrhundert zuvor verliehen hatte, um sie dem neulateinischen Epigramm anzunähern. Die galanten Lyriker des ausgehenden 17. Jahrhunderts verwenden die epigrammatische Struktur wieder, freilich in galanter, witzigerotischer Absicht.

\subsection{Die späte Bearbeitung Daniel Caspers von Lohenstein: Venus (1695)}

Die galante Lyrik des späten 17. Jahrhunderts bezieht sich nicht mehr auf Petrarca, sondern adaptatiert einen manieristisch übersteigerten Petrarkismus. In diesem Petrarkismus ızweiten Gradesı lassen sich einzelne Prätexte kaum noch isolieren, wie exemplarisch Daniel Casper von Lohensteins Venus zeigt.

Lohensteins mythologisches Epos Venus enthält einen 27 Verse umspannenden Passus (V. 1258-1284), der die pathetische Selbstdiagnose eines Liebenden präsentiert. ${ }^{41}$ Das liebende Ich diagnostiziert seine Liebe zu einer Roselinde in

Warümb bistu allein? Ich fühl allein das Band.

Wer mir nicht glauben wil/ der fang nur an und liebe.

Kreischt dich das Feuer nun so bleich/ so matt und trübe/

Warümb denn wirstu nicht zu Staub und Asch verbrant?

Ihr Eiß/ ihr kalter Sinn/ ihr Eiß/ ihr kaltes Wesen/

das hindert meine Gluth/ daß sie nicht kan genesen.

Das Feuer-Eis-Paradoxon aus Canz. 132, wird in der epigrammatischen Schlußpointe nicht mehr als unauflösliches Dilemma präsentiert, sondern auf Geliebte und Liebenden aufgeteilt.

40 Vgl. zu Schwan und Dichter die Studie von Michael Jakob: Schwanengefahr. Das lyrische Ich im Zeichen des Schwans. München und Wien 2000.

41 Daniel Casper von Lohenstein: Venus [ED: 1695]. In: Herm von Hoffmannswaldau und andrer Deutschen auserlesener und bißher ungedruckter Gedichter. Erster Theil. Hg. von Angelo George de Capua und Ernst Alfred Philippson. Tübingen 1961, S. 290-346, hier 328f.; wieder in Charlotte Brancaforte: Lohensteins Preisgedicht »Venus«. München 1974, S. 44f. und 66 (Werkstellenapparat). 
Krankheitsmetaphern, deren plurale Antithetik und uneingeleitete Bedingungssätze unverkennbar der Petrarca-Version des Martin Opitz nachgebildet sind. So variieren die antithetischen Versanfänge eines Reimpaars "Lieb' ich aus zwang" und "Lieb ich freywilliglich" (V. 1274f.) die Opitzischen Verse 6 und 7. Zweif elsfrei markiert den intertextuellen Bezug aber ein prägnantes Zitat in der Liebesklage:

Ists lieben? Liebe wird sich selber ja nicht hassen.

Ists haß? Haß wird uns wohl nicht so vereinigt lassen.

Ists hitze? freurt mich doch. Ists kälte? mir ist heiß.

Ich weiß nicht/ was ich will/ ich will nicht was ich weiß! (V. 1266-1269)

Lohenstein komprimiert in diesen zwei Reimpaaren das Opitzische Sonett, vor allem aber zitiert er die epigrammatische Pointe. Dabei vertauscht er die Reihenfolge, indem er die abstrakte Antimetabole dem antithetischen Temperaturvergleich nachordnet. Wie er den Temperaturvergleich noch antithetisch zuschärft, ist typisch für seine Parodie. Sie emphatisiert die petrarkistische Aussage, um deren klassische Dämpfung sich Opitz bemüht hatte. Die übertreibende Drastik verdankt Lohenstein aber einem zweiten petrarkistischen Mittelsmann: Giovan Battista Marino. Denn Marino hatte in der Liebesklage der Falsirena (Adone XII, 198-207) die dilemmatische Struktur von Petrarcas Sonett Nr. 132 amplifiziert und intensiviert:

-Ardo, lassa, o non ardo? Ahi qual io sento
stranio nel cor non conosciuto affetto?
Ė forse ardore? ardor non è, ché spento
l'avrei col pianto; è ben d'ardor sospetto.
Sospetto no, più tosto egli è tormento.
Come tormento fia, se dà diletto?
Diletto esser non può, poich'io mi doglio,
pur congiunto al piacer sento il cordoglio.
Or, se non è piacer, se non è affanno,
dunque è vano furor, dunque è follia.
Folle non è chi teme il proprio danno;
ma che pro se nol fugge, anzi il desia?
Forse amor? non amor. S'io non m'inganno,
odio però non è; che dunque fia?

Indem Lohenstein diese Liebesklage einer Frau einem männlichen Ich in den Mund legt, nähert er die Geschlechtsrollen an: >Liebe als Passion` ist eben nicht mehr geschlechtsspezifisch, sondern austauschbar. Die Klage von Lohensteins Rollen-Ich ist in Umfang, Argumentation, Metaphorik und syntaktischen Strukturen Marinos Petrarca-Parodie nachgebildet: Ein Fall zweischichtiger Intertextualität, wie er für die galante Lyrik der Zweiten Schlesischen Schule nicht untypisch ist: Lohenstein rezipiert den späthumanistischen Petrarkismus im Filter Marinos.

42 Giovan Battista Marino: Adone. Hg. von Giovanni Pozzi. Mailand 1988, hier XII 198, V. 1-XII 199, V. 6. 


\section{Zusammenf assung}

Martin Opitz hat in Konkurrenz zu neulateinischen Übertragungen Petrarcas Sonett Nr. 132 verdeutscht. Dieser aemulatio entsprechend ist seine PetrarcaVersion formal ssymmetrisiert<, epigrammatisch zugespitzt und das Pathos klassizistisch gedämpft. Sein Petrarkismus aus dem Geist des Späthumanismus wurde zum kanonischen Muster der Liebesdichtung, an dem sich viele Dichter des 17. Jahrhunderts versuchten. Noch zu Lebzeiten blieb das Muster nicht unwidersprochen, sondern wurde formal und inhaltlich variiert, bevor es um die Mitte des 17. Jahrhunderts in die grundsätzliche Kontroverse um Moral und ästhetische Autonomie geriet. Erst in der galanten Lyrik des Spätbarock diente das petrarkistische Mustersonett als Spielmaterial eines marinistisch überformten Petrarkismus. 